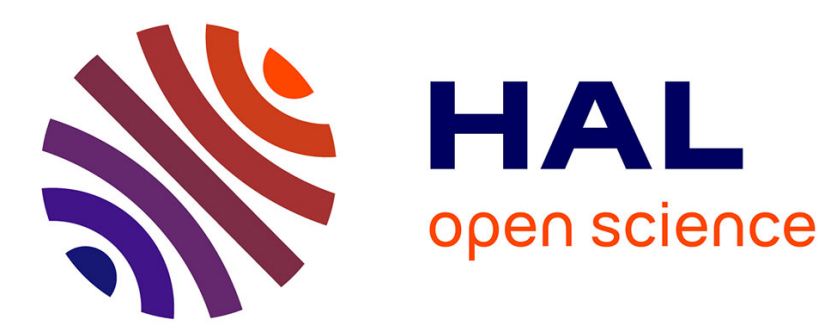

\title{
EIB y discurso político-educativo
}

David Abraham Macias Barres

\section{To cite this version:}

David Abraham Macias Barres. EIB y discurso político-educativo: ¿cómo se sitúan los cholos de la Costa?. ELAD-SILDA, 2019, Diglossie et bilinguisme en Équateur, 3. hal-02509574

\section{HAL Id: hal-02509574 https://hal.science/hal-02509574}

Submitted on 18 Mar 2020

HAL is a multi-disciplinary open access archive for the deposit and dissemination of scientific research documents, whether they are published or not. The documents may come from teaching and research institutions in France or abroad, or from public or private research centers.
L'archive ouverte pluridisciplinaire HAL, est destinée au dépôt et à la diffusion de documents scientifiques de niveau recherche, publiés ou non, émanant des établissements d'enseignement et de recherche français ou étrangers, des laboratoires publics ou privés. 


\title{
EIB y discurso político-educativo
}

\section{¿Cómo se sitúan los cholos de la Costa?}

\author{
David Macías Barrés \\ Université de Lyon (UJML3) \\ Centre d'Études Linguistiques - CEL EA 1663
}

david-abraham.macias-barres@univ-lyon3.fr

En Équateur, le discours étatique tend à minoriser - voire invisibiliser - l'identité chola. L'été 2018, Gilberto de la Rosa Cruz, président de la Fédération nationale du peuple cholo de l'Équateur (ou FENAPUCHODEC) - fondée en 2011 -, négocie avec l'État I'inclusion du peuple cholo dans l'article 56 de la Constitution actuelle, lequel mentionne les groupes ethniques reconnus par l'État. Le peuple s'auto-proclamant cholo espère ainsi bénéficier d'une plus grande visibilité et de la promotion de son identité à travers les politiques étatiques éducatives, culturelles et linguistiques. Nous présentons une étude lexicométrique qui nous permet d'évaluer le degré de visibilité du peuple cholo du littoral par rapport à d'autres groupes humains de l'Équateur. Nous nous penchons notamment sur le discours politico-éducatif, en tant que reflet du discours étatique, présent dans les manuels scolaires (libros de texto), lorsque ceux-ci définissent, à l'attention des élèves, I'organisation politique, sociale et administrative de l'État. Étant donné que les Cholos sont un peuple hispanophone, nous observons le système d'Éducation nationale (enseigné en espagnol) et analysons le discours qui se dégage des manuels scolaires des trois matières du Baccalauréat général unifié (ou BGU) : (a) Éducation citoyenne, (b) Histoire et (c) Langue et littérature.

Mots-clés : Équateur, Cholos, littoral, discours étatique, discours national, discours politico-éducatif, identité culturelle et linguistique, patrimoine, groupe minorisé

En Ecuador, el discurso estatal tiende a minorizar -o incluso invisibilizar- la identidad chola. En el verano del 2018, Gilberto de la Rosa Cruz, presidente de la Federación Nacional del Pueblo Cholo del Ecuador (o FENAPUCHODEC) - fundada en el 2011-, negocia con el Estado la inclusión de dicho pueblo en el artículo 56 de la Constitución vigente, artículo en el que se mencionan los grupos étnicos reconocidos por el Estado. Así, este pueblo, que se autoproclama cholo, espera conseguir una mayor visibilidad y la promoción de su identidad a través de las políticas estatales educativas, culturales y lingüísticas. Presentamos un estudio lexicométrico que nos permite evaluar el grado de visibilidad del pueblo cholo costeño con respecto a los demás grupos humanos de Ecuador. Nos centramos particularmente en el discurso político-educativo, reflejo del discurso estatal, presente en los manuales escolares (o libros de texto), cuando estos definen, para el alumnado, la organización política, social y administrativa del Estado. Como los cholos son un pueblo hispanohablante, nos focalizamos en el Sistema de Educación Nacional (enseñado en español) y analizamos el 
discurso que se desprende de los manuales escolares de cuatro tres materias en el programa del Bachillerato General Unificado (o BGU): (a) Educación para la ciudadanía, (b) Historia y (c) Lengua y Literatura

Palabras claves: Ecuador, cholos, litoral, discurso estatal, discurso nacional, discurso político-educativo, identidad cultural y lingüística, patrimonio, grupo minorizado

In Ecuador, State Discourse tends to minorize -or even invisibilize- cholo identity. In summer 2018, Gilberto de la Rosa Cruz, President of the National Federation of Cholo People in Ecuador (or FENAPUCHODEC) -founded in 2011-, negotiates the inclusion of Cholos in the article $n^{\circ} 56$ of the actual Ecuadorian Constitution with the government; the article that mentions the ethnic groups recognized by the Ecuadorian government. By doing so, this pueblo (people in Spanish), that auto-proclaims itself Cholo, hopes to gain more visibility and to guarantee the promotion of their identity through public education, cultural, and language policies. By means of a lexicometrical study, I intend to show the degree of visibility of littoral Cholo people compared to the other human groups in Ecuador. I will center mainly on Political-Educational Discourse, which reflects State Discourse, found in those textbooks (libros de texto) that define the political, social and administrative organization of the State. Since Cholos are Spanish-speakers, I only use textbooks of three subjects coming from the General Unified Baccalaureate Program (GUB program) of the National Education System (taught in Spanish): (a) Civic Education, (b) History, and (c) Language and Literature.

Keywords: Ecuador, Cholos, littoral, State Discourse, National Discourse, Political-Educational Discourse, cultural and linguistic identity, heritage, minorized group

\section{Introducción}

Como lo recalca M. Rodríguez Cruz [2018], en Ecuador coexisten dos sistemas educativos: el Sistema de Educación Nacional para los hispanohablantes y el Sistema de Educación Intercultural Bilingüe pensado para las nacionalidades indígenas. Como se puede constatar, esta bipolarización del sistema educativo se realizó a partir del idioma del público meta. Pero esta división parece no tener en cuenta los matices que pueden existir con respecto a las especificidades culturales y lingüísticas que caracterizan a las nacionalidades y pueblos del Ecuador. Y es que, como ya lo indicamos en D. Macías Barrés [2014: 3], proponemos una diferencia entre nacionalidades y pueblos. A nivel lingüístico, la pertenencia a una nacionalidad parece basarse en el idioma -o la lengua- de la comunidad (e.g. nacionalidad kichwa, nacionalidad shuar, etc.). Sin embargo, la pertenencia a un pueblo parece efectuarse a través del habla que comparte la comunidad (e.g. los pueblos saraguro y chibuleo forman parte de la nacionalidad kichwa) y no el idioma. Entiéndase idioma como un conjunto de signos y reglas utilizadas por los locutores de esta; en cambio, habla es el empleo de tal sistema por un hablante y por una comunidad lingüística. Dicho de otra manera, como lo precisamos en D. Macías Barrés [2014: 4], "los pueblos de una misma nacionalidad utilizarán la misma lengua, pero el uso que hace cada pueblo de ella se denominará habla". Los pueblos son, por consiguiente, un subgrupo de la nacionalidad. Entonces, en Ecuador, la situación sociolingüística es bastante 
compleja tanto más cuanto que los términos utilizados para estudiarla son aún en la actualidad poco precisos y pueden variar de un autor a otro, como es el caso de nacionalidad y pueblo. En D. Macías Barrés [2014: 4] incluso sometimos la hipótesis que se debería considerar que existe una nacionalidad hispanohablante en la que existen varios pueblos, uno de ellos sería el pueblo cholo de la Costa.

Dentro de un proceso que D. Bauer [2010] denomina reindigenización ${ }^{1}$, a partir del año 2001 el pueblo cholo ha decidido poner de relieve su patrimonio ancestral, en particular el manteño-huancavilca, y así ponerse a la par de las nacionalidades indígenas para exigir que se respeten sus derechos comunales. De conseguirlo, serían considerados indígenas costeños como lo sugiere S. Álvarez Litben [2016: 327]. Y es que, para la autora, se trata de un pueblo ancestral que ha tenido que adaptarse a los diferentes gobiernos que han regido el territorio que actualmente se conoce como República del Ecuador. Ahora bien, D. Bauer [2010: 179] indica que para el Estado ecuatoriano, estos pueblos son más bien considerados como mestizos -o incluso montuvios ${ }^{2}$ - porque no hablan un idioma ancestral. El pueblo cholo parece ser minorizado o incluso invisibilizado por el discurso estatal, en particular el que se desprende de las políticas educativas.

Como los cholos son un pueblo hispanohablante, analizaremos el discurso procedente de los textos del Sistema de Educación Nacional o hispano, que es el que se aplica a estas poblaciones costeras. En esta contribución analizaremos el discurso estatal con respecto al pueblo cholo del Ecuador, en particular el político-educativo presente en los manuales escolares 0 libros de texto. A partir de los mismos, haremos un estudio lexicométrico. Contabilizaremos el número de ocurrencias del lexema chol- frente al número de ocurrencias de los lexemas que hacen alusión a los demás grupos étnicos en Ecuador. Así, esperamos evaluar el grado de visibilidad de este pueblo en el discurso educativo, especialmente el político-educativo. Con dicho fin, en este artículo, primero abordaremos las diferentes etnicidades de la Costa ecuatoriana, centrándonos en la cultura chola. Luego, contrastaremos la presencia del cholo en el discurso regional frente al discurso nacional y estatal. Finalmente, presentaremos el estudio lexicométrico, al que nos referimos anteriormente, realizado a partir de manuales escolares del Bachillerato General Unificado o BGU, consultables en el sitio web del Ministerio de Educación del Ecuador.

1 D. Bauer [2010] propone el término reindigenización, también conocido como etnogénesis (cf. S. Álvarez Litben, 2016). Se trata de un proceso a través del cual ciertos pueblos, considerados como minorías (i.e. minorizados), se apoyan en su patrimonio histórico para reactivar ciertos rasgos étnicos suyos, invisibilizados por el grupo cultural dominante.

2 En Ecuador coexisten dos grafías: montubio y montuvio. El pueblo montuvio del Ecuador prefiere la segunda grafía. En efecto, El Diccionario de la Lengua Española (o DLE) define montubio como una persona "montaraz y grosera", definición con a la que no adhieren. En cambio, se define montuvio como un ecuatorianismo que significa "campesino de la costa". Eso explica su preferencia por este último. Para saber más sobre el montuvio costeño, ver. D. Macías Barrés [2014]. 


\section{Etnicidades de la Costa ecuatoriana y el cholo de la Costa}

A nivel jurídico, el artículo 56 de la Constitución del 2008 indica: "Las comunidades, pueblos y nacionalidades indígenas, el pueblo afroecuatoriano, el pueblo montubio y las comunas forman parte del Estado ecuatoriano, único e indivisible" ${ }^{\prime \prime}$. Se reconoce, entonces, la existencia de los siguientes grupos étnicos: indígenas (o pueblos ancestrales), afrodescendientes (o afroecuatorianos) y montuvios (escrito "montubio" en la Constitución). Podemos ver que la Carta Magna no reconoce la existencia del pueblo cholo. Sin embargo, este pueblo se ha organizado $y$, en particular en el verano 2018 (julio-agosto), entabla negociaciones con el Estado para que la Asamblea Nacional del Ecuador los reconozca, tal como lo indica N. Palma [2018a; 2018b]. Estos pueblos de la costa tratan de reactivar su patrimonio ancestral (proceso llamado, como ya lo mencionamos, reindigenización o etnogénesis) para ser asociados a "indígenas hispanohablantes". En efecto, como lo indica C. Dueñas [1997; 2010], al adaptarse a los diferentes regímenes políticos, los cholos perdieron su lengua ancestral, cediendo al proceso de hispanización.

Ahora bien, como lo subrayamos en D. Macías Barrés [2019: 3], en el Ecuador, para el imaginario colectivo y el discurso nacional, los indígenas se encuentran o bien en la Sierra o bien en la Amazonía. Como lo indica K. Smith [2015: 21], el discurso dominante ha borrado prácticamente a los pueblos ancestrales de la Costa. Así, pues, en la Costa ecuatoriana, solo ciertos grupos étnicos han conseguido ser reconocidos por el Estado y, por ende, gozan de una mayor visibilidad, como los afroecuatorianos (p. ej. en Esmeraldas) y los montuvios (en los campos de la provincia del Guayas, Manabí y El Oro). El pueblo cholo no ha conseguido dicho reconocimiento jurídico, tal vez porque se pensó que el término "comunas" bastaría. En el 2001, el incidente de la comuna Macaboa ${ }^{4}$, en el sur de la provincia de Manabí, parece haber marcado un hito en la construcción identitaria de estos pueblos. En ese año, el Ministerio de Agricultura y Ganadería autorizó la venta de 34 hectáreas de tierras comunales a un inversor ecuatoriano-suizo. Para dicho ministerio, la venta fue legal por tratarse de un pueblo mestizo y no de un pueblo indígena. Como lo sugiere D. Bauer [2010: 103], fue un momento clave para la construcción identitaria del pueblo cholo, ya que le pareció relevante reactivar su patrimonio ancestral, i.e. reindigenizarse. A pesar de esto, S. Álvarez Litben [2016: 338] resalta que, al momento de

3 En la Constitución, el término "montubio" se encuentra escrito con " $b$ ". Nosotros hemos preferido utilizar más bien la grafía con " $v$ ", i.e. "montuvio" que es con el que la misma comunidad se autoidentifica.

4 D. Bauer [2010] prefirió utilizar un nombre inventado para hacer alusión a una de las comunas que forman parte de la parroquia Salango, cantón Puerto López. 
redactar la Constitución en el 2007 , la identidad chola no les quedaba clara a los asambleístas ajenos a esta región e incluso no sabían que había que otorgarles derechos comunales.

Parece que la identidad chola no está claramente definida, tanto a nivel cultural como lingüístico. Como lo señalamos en D. Macías Barrés [en prensa], los marcadores étnicos de los cholos a veces se confunden con los de los montuvios, e.g. las danzas folklóricas ${ }^{5}$ parecen asemejarse. Incluso a nivel lingüístico, como ya lo subrayamos en D. Macías Barrés [2019: 3], contrariamente a lo que ocurre con los montuvios que tienen el amorfino ${ }^{6}$, las manifestaciones lingüísticas del pueblo cholo son poco conocidas $y$, en todo caso, no son estudiadas en el plan curricular del sistema educativo público. El Diccionario de la lengua española o DLE [2019] establece que se trata de un significante polisémico. Efectivamente, dos acepciones son propuestas por el diccionario:

(a) Mestizo de sangre europea e indígena.

(b) Dicho de un indio: Que adopta los usos occidentales.

Como podemos observar, ambas contienen matices distintos. En la primera, se hace alusión a un mestizo; mientras que, en la segunda, se hace alusión a un indígena. Sin embargo, podemos resaltar que ambas tienen en común el componente indígena y la mezcla con el componente europeo occidental. En la primera definición, se hace alusión a un mestizaje más bien étnico (mezcla racial) mientras que, en la segunda, se trata de un mestizaje cultural (o incluso del aculturamiento del indígena). A esto cabe añadir que, en función de la región geográfica, cholo no tendrá el mismo referente, no sólo a nivel panhispánico sino también dentro del mismo Ecuador. Como lo indica E. Sinardet [2010], si bien a nivel nacional cholo significa mestizo, también se utiliza a nivel local para designar a un indígena. La autora señala que se opone cholo (i.e. indio de la Costa) a longo (i.e. indio de la Sierra).

Además, en Ecuador, como lo señalan K. Roitman \& A. Oviedo [2017], cholo se utiliza de manera peyorativa. Según los autores, de manera generalizada y basándose en entrevistas realizadas tanto en Guayaquil como en Quito, indistintamente del estrato socioeconómico, el término cholo se utiliza de manera despectiva y para menospreciar -y hasta denigrar- algo o a alguien. Incluso, tanto en Ecuador como en la región andina, se ha creado el verbo cholear que, según el DLE [2019], significa "tratar a alguien despectivamente". Esta connotación negativa se ve plasmada, también, en la televisión ecuatoriana. En la pantalla chica, el cholo es casi siempre

5 Basta con comparar los siguientes vídeos de sus danzas respectivas en la plataforma Youtube: danza chola (https://youtu.be/N-WsZomcGkU) vs danza montuvia (https://youtu. be/6tF7yKO5WfQ).

6 El amorfino es una creación poético-musical, acompañada de una danza, que se asocia casi exclusivamente al pueblo montuvio (cf. D. Macías Barrés, 2014). 
representado de manera estereotipada y con fines cómicos. En los años 80, una comedia de situación llamada Mis adorables entenados hizo popular la frase: "iQué cholo!". Y es que, Felipe -uno de los personajes- la utilizaba para denigrar a sus hermanastros por no ser tan blancos como él y, en particular, por no haber adoptado las costumbres de la élite guayaquileña blanco-mestiza. A partir del 2000, personajes televisivos como El cholito o Escholita Solitaria, ambos del comediante David Reinoso [Wikipedia, 2019], muestran al cholo como alguien de rasgos indígenas o de piel más oscura que el ideal blanco del discurso dominante. También se lo muestra como alguien vulgar, menos culto o ingenuo. Entonces, en el imaginario colectivo, cholo no se asocia a una visión positiva del patrimonio ancestral ni del mestizaje.

Los cholos de la Costa, en su lucha para tener una mejor visibilidad -a nivel nacional e internacional-, se han apropiado de este significante para autodefinirse. Como ya lo indicamos, buscan reactivar su patrimonio ancestral a través de la reindigenización. De esta forma, su reivindicación social se ha vuelto étnica y su discurso -en particular en el ámbito político- ha adquirido tintes étnicos, tal como el discurso político indígena. La reindigenización de su patrimonio -en particular el inmaterial (e.g. su discurso y sus prácticas sociales)- les ha permitido ponerse a la par de los pueblos indígenas para negociar con el Estado y exigir el respeto de sus derechos.

\section{Los cholos en el discurso nacional y estatal}

A inicios del siglo xx, más precisamente a partir de los años 30, el Grupo de Guayaquil en la Costa se interesó en mostrar nuevas representaciones de la ecuatorianidad a través del discurso literario. Estas representaciones salían del modelo decimonónico, i.e. la supervaloración de lo europeo, e hicieron visibles nuevas identidades como la montuvia (bajo la pluma de José de la Cuadra) y la chola (en las obras de Demetrio Aguilera Malta). Entre los años 30 y 50, las obras de Demetrio Aguilera Malta, inscritas dentro del realismo social, denunciaron la situación de explotación y de enajenación cultural de los cholos ante la imposición de nuevas formas de economía, e.g. en Don Goyo (1933) se reemplaza la pesca artesanal por la tala del manglar impuesta por los comerciantes de Guayaquil. Como ocurriera con los montuvios, este discurso literario brindó cierta visibilidad a la cultura chola ya en aquella época. Sin embargo, como lo indicamos en D. Macías Barrés [en prensa], al parecer esta identidad ha quedado estancada a escala regional y ha tenido poco impacto en el discurso nacional.

K. Smith [2015: 13], antropóloga que estudia al pueblo cholo en la actualidad, sostiene que el discurso es importante porque permite establecer de manera bastante fija la manera cómo las categorías sociales son percibidas por el conjunto del país y lo que se considera dentro de la "normalidad". Para K. Smith [2015: 2], en Ecuador, la geografía está "racializada" (racialized geography), lo que implica que el imaginario colectivo y, por ende, el discurso 
nacional esperan que ciertos grupos humanos se encuentren en ciertas regiones del país y no en otras. Se supone, entonces, que los indígenas están en la Sierra o en la Amazonía y no en la Costa. La autoproclamación por una parte de este pueblo costeño como "indios costeños" o "pueblo cholo" crea disonancia cognitiva en el resto de la población, i.e. estado de tensión en el que se encuentran los individuos confrontados a una situación contraria a sus convicciones, a su manera de actuar o de pensar. Tal vez este recurso forme parte de un proceso de empoderamiento y sea una estrategia que les permitiría integrar el discurso estatal a mediano y largo plazo, en particular el político-educativo.

En efecto, el discurso estatal goza de varios medios de difusión. Las políticas estatales permiten dar a conocer este discurso y promoverlo. En nuestro análisis distinguimos discurso político, educativo y político-educativo. Para P. Fabbri \& A. Marcarino [2002: 18], el discurso político es:

un discurso de campo destinado a llamar y a responder, a disuadir y a convencer; un discurso de hombres para transformar hombres y relaciones entre los hombres, no sólo un medio para re-producir lo real.

Dicho de otra manera, pensamos que el discurso político, en este caso el estatal, tiene una función perlocutiva: transformar hombres (i.e. construir una identidad nacional) y crear relaciones (i.e. asegurar la cohesión nacional). Por otro lado, para nosotros, el discurso educativo -que también se deprende del discurso estatal y que, por ende, es un reflejo de este- es el que promueven los docentes de la Educación Pública y se encuentra en los textos que rigen las políticas educativas estatales así como los materiales utilizados, e.g. los manuales escolares.

Para nuestro análisis, entendemos por discurso político-educativo, una rama del discurso estatal que se desprende de los manuales escolares y que concierne a la organización política, social y administrativa del Estado. Este discurso fomentado por las políticas educativas hace que se vehicule el discurso estatal con respecto a la organización social y, de esta forma, que ciertos grupos étnicos sean reconocidos. El análisis del discurso políticoeducativo nos permitirá evaluar el grado de visibilidad de los grupos humanos en el Ecuador. Como ya lo habíamos evocado, para el discurso dominante y el imaginario colectivo, en la Costa parece no haber amerindios. En el artículo 56 de la Constitución, además de las comunidades, pueblos y nacionalidades indígenas, solo dos grupos étnicos han sido reconocidos oficialmente en la región litoral: los afroecuatorianos y los montuvios. En el mismo artículo, se mencionan las comunas. En su libro De Huancavilcas a comuneros. Relaciones interétnicas en la Península de Santa Elena (Ecuador), S. Álvarez Litben [2001] ha preferido el término comuneros para referirse a los cholos de la Costa, tal vez por la connotación negativa de este último.

7 Las cursivas son del autor. 
Y es que, en la Costa, estos pueblos se han organizado en comunas. Esta organización político-administrativa parece ser la que mejor cuajó con su cosmovisión y cultura locales, basadas -entre otros- en la administración y posesión colectiva de bienes y tierras. Según el artículo 1 de la Ley de Organización y Régimen de Comunas o LORC [2004], las comunas son:

[T]odo centro poblado que no tenga categoría de parroquia, que existiera en la actualidad o que se estableciere en lo futuro, y que fuere conocido con el nombre de caserío, anejo, barrio, partido, comunidad, parcialidad, o cualquier otra designación, llevará el nombre de comuna, a más del nombre propio con el que haya existido o con el que se fundare.

Para constituir una, se necesita que el número de habitantes no sea menor de cincuenta (artículo 5). Los habitantes pueden poseer bienes colectivos, en particular tierras (e.g. de labranza y de pastoreo). Como pasa con cholo, el término comuna es poco definitorio. A priori cualquier grupo humano de cincuenta personas, en cualquier lugar del país, ocupando un territorio dado, puede establecer una comuna. No obstante, ¿el hecho de ser una comuna les garantizará el reconocimiento y el respeto de sus derechos por parte del Estado? En diciembre del 2001, la venta de tierras pertenecientes a la comuna Macaboa, en la Costa ecuatoriana, fue autorizada y aceptada por el gobierno ecuatoriano. Como ya lo indicamos, según D. Bauer [2010: 79], el Ministerio de Agricultura y de Ganadería, del que dependía dicha comuna, sostuvo que la venta era legal porque los habitantes de Macaboa no eran indígenas sino mestizos o montuvios. Este incidente muestra cómo los derechos comunales no son plenamente respetados, dependiendo de cómo se interpretan las leyes. Así, ya sea bajo el término cholo o comunero, parece que este pueblo se encuentra virtualmente invisibilizado en el discurso estatal y en particular en el político-educativo, como lo veremos a continuación.

\section{Discurso político-educativo y manuales escolares}

El análisis de manuales escolares -o libros de texto- del sitio del Ministerio de Educación del Ecuador (cf. https://educacion.gob.ec/libros-de-texto/) nos permite evaluar el grado de visibilidad del pueblo cholo con respecto a los demás grupos étnicos en el Ecuador, excluyendo de nuestro estudio el grupo blanco-mestizo ${ }^{8}$. Como ya lo especificamos, dado que los cholos son hispanohablantes, nos centraremos en el Sistema de Educación Nacional o hispano, que es el que se aplica a estas comunas. Hemos escogido tres

8 Como el grupo blanco-mestizo ha sido históricamente el dominante, nos hemos centrado en los grupos étnicos minorizados desde la Colonia. Concretamente, en este estudio, se trata de mostrar la poca o casi nula visibilidad que se le brinda a los cholos de la Costa en el discurso estatal. 
materias del Bachillerato General Unificado (BGU) 9 : (a) Educación para la ciudadanía, (b) Historia y (c) Lengua y literatura. Estas nos parecieron pertinentes por ser las que participan en el desarrollo del sentimiento de pertenencia a la nación.

Aunque nos centraremos en el lexema chol-, hemos hecho un inventario del número de ocurrencias de los lexemas que se refieren a los demás grupos étnicos reconocidos en el artículo 56 de la Constitución: indígen(para hacer alusión los amerindios), afro- (con los afroecuatorianos como referente) y montuv-, montub- o incluso amorfino (para referirse al universo montuvio). De esta manera, compararemos su presencia a nivel cuantitativo con respecto al significante cholo. Luego, nos interesaremos en el co(n)texto de aparición de dicho significante para evaluar si se trata de un término genérico o específicamente de los cholos de la Costa.

\subsection{Educación para la ciudadanía}

La materia Educación para la ciudadanía está prevista para 1ero y 2do curso del BGU. El cuadro 1 infra muestra que se hace muy poca alusión a los cholos y a su cultura, en particular comparado con los indígenas. A nivel cuantitativo, la presencia del lexema chol- es claramente inferior con respecto a los otros lexemas. $Y$ es que, con respecto al número total de ocurrencias de todos los lexemas, en 1er curso el lexema chol- solo representa un $0,71 \%$ del conjunto y en 2 do un $2,86 \%$. A nivel cualitativo, es difícil evaluar si el significante cholo alude al significado "cholo de la Costa". Podría referirse, más bien, a cualquier tipo de mestizo, uno de los referentes de dicho significante. En todo caso, descartamos que haga referencia al significado indígenas por la presencia cercana del signo indígena en el cotexto, tal como lo vemos en los enunciados (1)-(8), ya que sería demasiado redundante. Así, en esta materia, concluimos que se hace muy poca alusión a los cholos, ya sean estos de la Costa o de la Sierra.

\section{Cuadro 1: BGU, Educación para la ciudadanía}

\begin{tabular}{|c|c|c|}
\hline & $1^{\text {er }}$ curso & $2^{\text {do }}$ curso \\
\hline indígen- & 110 & 103 \\
\hline afro- & 13 & 69 \\
\hline montu(b-/v-) o amorfino & 15 & 65 \\
\hline chol- & 1 & 7 \\
\hline
\end{tabular}

9 El Bachillerato General Unificado (BGU) corresponde a los tres últimos años de educación antes de ingresar a la universidad. Las materias del BGU forman parte de un tronco común ya que los alumnos pueden escoger dos especialidades, i.e. bachillerato en ciencias y bachillerato tecnológico. 


\section{$1^{\text {er }}$ curso}

(1) La clase criolla estaba conformada por terratenientes, dueños de la tierra y bienes, quienes subordinaron a grupos como artesanos y pequeños propietarios, indígenas, cholos, montubios y afrodescendientes, que eran la mayoría.

\section{$2^{\text {do }}$ curso}

(2) Con el aparecimiento del Estado, los mestizos lograron acceder al poder político, mucho más con la Revolución liberal, que reconoció la ciudadanía para todos: hombres, mujeres, indios, negros, cholos, mulatos, mestizos y blancos.

(3) Comenten si saben de personas que han negado a sus padres, abuelos o familiares porque son indígenas, afros, cholos, extranjeros.

(4) Las mujeres, los niños y adolescentes, los indígenas, afrodescendientes, montubios y cholos de la época, y mestizos o blancos pobres no eran ciudadanos.

(5) La Constitución 2008 reconoce la ciudadanía a: a. Los niños, niñas y adolescentes $b$. Cholos, afros, indígenas, montubios, mestizos y blancos c. Personas de opción sexual diversa d. Todas las anteriores.

(6) La Constitución 1830 reconocía la ciudadanía a: a. Los hombres con dinero b. Las mujeres, indígenas, afros c. Cholos y montubios mayores de 21 años d. Blancos y mestizos sin dinero.

(7) La Constitución 1861 reconoce la ciudadanía a: a. Los indígenas, afros, montubios b. Hombres mayores de 21 años, casados y que saben leer y escribir c. Hombres y mujeres mayores de 21 años, casados y que saben leer y escribir $d$. Cholos y montubios que saben leer y escribir.

(8) La Constitución 1906 reconoce la ciudadanía a: a. Hombres y mujeres mayores de 21 años, que sepan leer y escribir b. Mestizos y blancos que saben leer y escribir c. Indígenas, montubios, cholos, afros d. Hombres y mujeres mayores de 21 años con propiedades.

\subsection{Historia}

La materia Historia se enseña en 1ero, 2do y 3er año del BGU. En el cuadro 2 infra, vemos que esta materia, que retoma los eventos que dieron lugar a la formación de la nación ecuatoriana y que permitiría asegurar la cohesión nacional bajo un discurso de la memoria nacional, no menciona ni a los cholos ni a los montuvios. En esta materia, ambos grupos étnicos están invisibilizados en el discurso que se deprende de los manuales escolares del BGU. 


\section{Cuadro 2: BGU, Historia}

\begin{tabular}{|l|r|r|r|}
\hline & \multicolumn{1}{|c|}{$\mathbf{1}^{\text {er }}$ curso } & \multicolumn{1}{|c|}{$\mathbf{2}^{\text {do }}$ curso } & \multicolumn{1}{|c|}{$3^{\text {er }}$ curso } \\
\hline indígen- & 4 & 38 & 155 \\
\hline afro- & 2 & 1 & 33 \\
\hline montu(b-/v-) o amorfino & 0 & 0 & 0 \\
\hline chol- & 0 & 0 & 0 \\
\hline
\end{tabular}

\subsection{Lengua y literatura}

Lengua y literatura se enseña también en los tres años del BGU. A nivel cuantitativo, con cifras parecidas a las de los lexemas que se refieren al universo montuvio, la alusión a los cholos es minoritaria de nuevo. En 1er año es incluso nula mientras que en 2 do y $3 e r$ año corresponde respectivamente al $18,18 \%$ y al $8,33 \%$ del conjunto de lexemas. A nivel cualitativo, al analizar los enunciados (9)-(14), constatamos que casi todas las ocurrencias de chol- tienen como referente a los cholos de la Costa ${ }^{10}$. En efecto, se hace alusión a los cholos/personajes de la obra de Demetrio Aguilera Malta quien, como ya lo mencionamos, escribió sobre ellos principalmente entre los años 30 y 50 .

\section{Cuadro 3: Lengua y literatura}

\begin{tabular}{|l|r|r|r|}
\hline & \multicolumn{1}{|c|}{$\mathbf{1}^{\text {er }}$ curso } & $2^{\text {do }}$ curso & \multicolumn{1}{|c|}{ 3er $^{\text {curso }}$} \\
\hline indígen- & 17 & 14 & 17 \\
\hline afro- & 0 & 0 & 2 \\
\hline montu $(b-/ v-)$ o amorfino & 0 & 4 & 3 \\
\hline chol- & 0 & 4 & 2 \\
\hline
\end{tabular}

\section{$2^{\text {do }}$ curso}

(9) El Cholo Que Odio la Plata11

(10) Los dos eran cholos.

(11) No eran malos los cholos.

(12) El cholo que odió la plata

10 Hemos excluido el enunciado 13 que presenta el significante fuera de contexto y como un disfemismo.

11 Hemos retomado el enunciado tal cual está escrito en el texto, aunque no adhiramos a las normas tipográficas. 


\section{$3^{\text {er }}$ curso}

(13) Analiza por qué son disfemismos los siguientes términos: carischina, mandarina, longo, cholo, bagre, chagra.

(14) Colaboró con Enrique Gil Gilbert y Joaquín Gallegos Lara en un libro colectivo de cuentos que ganó un lugar apreciado en nuestra literatura: Los que se van. Cuentos del cholo y del montuvio.

Vemos, pues, que en el discurso educativo, y en particular el políticoeducativo, se hace muy poca alusión al pueblo cholo. La materia Educación a la ciudadanía utiliza el significante cholo diferenciándolo de indígena; sin embargo, no se especifica claramente cuál es el referente ni tampoco se da una definición de lo que es. En la materia Historia, ni siquiera se menciona al cholo, ya sea de la Costa o de la Sierra, como parte integrante del Ecuador ni como actor dentro de los eventos históricos nacionales. En cambio, en Lengua y literatura, la alusión a los cholos costeños, aunque minoritaria como sucede con los montuvios, es clara ya que son los personajes del escritor Demetrio Aguilera Malta en sus obras y cuentos.

Entonces, el lexema chol- tiene muy pocas ocurrencias comparado con los demás, especialmente con el lexema indígen-. Esta población costera no goza entonces del mismo grado de visibilidad que los indígenas. Los cholos se encuentran minorizados en el discurso estatal, el educativo y, más concretamente, el político-educativo. Así, su promoción cultural por el Currículo estatal parece ser menor, si se compara con los demás grupos étnicos.

\section{Conclusión}

Contrariamente a lo ocurrido con el montuvio, la identidad chola no se ve suficientemente reflejada en el discurso estatal. En el discurso políticoeducativo, tanto a nivel cuantitativo como cualitativo, constatamos que las alusiones a este pueblo son inferiores con respecto a los demás grupos étnicos, en particular los indígenas. Así, las comunas costeras, que se han autoproclamado cholas, han decidido reivindicar su patrimonio ancestral con el fin de ser asociadas a indígenas costeños, no a mestizos ni a montuvios. A nivel discursivo, se han (re)apropiado estratégicamente de un término de connotación peyorativa y se han inspirado del discurso indigenista serrano para tener una mejor -e incluso mayor- visibilidad a nivel político. Esto les permite exigir que se respeten sus derechos comunales y que estos no pasen después de los intereses del grupo blanco-mestizo.

Su autodefinición como cholos o indígenas costeños crea disonancia cognitiva a varios niveles. Primero, porque en el imaginario colectivo nacional, la presencia indígena en la Costa es prácticamente inexistente. Segundo, 
porque, como lo sugiere K. Smith [2015: 37], el Estado considera que los indígenas tienen que hablar un idioma ancestral y, de no ser así, son más bien considerados mestizos. Como ya lo mencionamos, en esto se ha basado la política educativa pública. Existen dos sistemas de Educación Pública: uno hispano en castellano y otro indígena en lengua indígena/ancestral. Si los cholos son indígenas costeños, ¿qué sistema de educación habría que aplicar? Los cholos -como indígenas hispanohablantes- no entrarían, pues, dentro de las delimitaciones definidas por el discurso estatal.

Así, al analizar el discurso político-educativo, constatamos que los cholos no gozan -al menos todavía- de la misma visibilidad que los demás grupos de la Costa ecuatoriana. En la actualidad, casi excluidos del discurso estatal, su patrimonio cultural es muy poco promovido por el Estado-nación y, por ende, poco valorizado dentro del paisaje cultural y lingüístico del país. Por el momento, la salvaguarda y preservación del patrimonio cultural cholo no forma parte de las políticas públicas.

A pesar de los esfuerzos emprendidos estos últimos años, en particular desde el 2008 con la Constitución vigente, el sistema educativo ecuatoriano aún no ha conseguido tomar en cuenta plenamente la diversidad cultural existente en el país, en términos de contenido (i.e. se estudia muy poco la cultura chola en el Currículo) y de público meta (i.e. ¿se puede concebir la existencia de indígenas que no hablen un idioma ancestral?). Podemos, entonces, constatar que la bipolarización del sistema educativo público ecuatoriano (grosso modo hispano vs indígena) no se aplicaría a estos pueblos. En efecto, ni en el sistema hispano ni en el indígena, se estudiaría suficientemente su propia cultura: la chola.

Para nosotros, un sistema educativo central (top-down), como el sistema actual, debería ser completado por un sistema educativo provincial y/o regional (bottom-up), i.e. en el cual el público meta sería consultado. El sistema central permitiría inculcar valores nacionales y la pertenencia a la nación ecuatoriana. El sistema provincial/regional valorizaría la cultura local insertándola dentro del paisaje cultural y lingüístico del Estadonación. Esto permitiría: a) inventariar y tener en cuenta las particularidades locales, b) poner de realce las manifestaciones culturales y lingüísticas de los diferentes pueblos que habitan el Ecuador -hispanohablantes o no-, c) evitar que la Educación Pública (y el discurso que se desprende de esta) sea percibida como una imposición. Así, el sistema educativo nacional podría hacer resaltar su dimensión intercultural transversal, al ser respetuosa de todas las culturas del Ecuador y de todas las manifestaciones de estas. 


\section{Bibliografía}

Álvarez Litben Silvia Graciela, 2001 [1999], De Huancavilcas a comuneros: relaciones interétnicas en la península de Santa Elena - Ecuador, Quito: Editorial AbyaYala.

Álvarez Litben Silvia Graciela, 2016, "La importancia de tener nombre: identidad y derechos territoriales para las comunas de Santa Elena, Ecuador", Revista de Antropología Experimental, n. ${ }^{\circ} 16,325-352$, disponible en https://revistaselectronicas.ujaen.es/index.php/rae/article/ view/2896/2517

Asociación de Academias de la Lengua Española \& Real Academia Española, 2019, "cholear", Diccionario de la lengua española, disponible en https:// dle.rae.es/?id=8xZwkbJ

Asociación de Academias de la Lengua Española \& Real Academia Española, 2019, "cholo, la", Diccionario de la lengua española, disponible en http:// dle.rae.es/?id=EMIPbqI

Bauer Daniel, 2010, "Re-articulating Identity: The Shifting Landscape of Indigenous Politics and Power on the Ecuadorian Coast", Bulletin of Latin American Research, n. ${ }^{\circ} 29$ (2), 170-186, disponible en https://onlinelibrary.wiley.com/doi/abs/10.1111/j.1470-9856.2009.00347.x

Colaboradores de Wikipedia, 2018, "David Reinoso", Wikipedia, la enciclopedia libre, disponible en https://es.wikipedia.org/wiki/David_Reinoso

Colaboradores de Wikipedia, 2018, "Mis adorables entenados", Wikipedia, la enciclopedia libre, disponible en https://es.wikipedia.org/w/index.php?title=Mis_adorables_entenados\&oldid $=109442436$

Congreso Nacional del Ecuador, 2004 [1937], Ley de organización y régimen de comunas, Quito: s.ed., disponible en https://www.acnur.org/fileadmin/Documentos/BDL/2008/6614.pdf?view=1

Dueñas Carmen, 1997, Marqueses, cacaoteros y vecinos de Portoviejo: cultura política en la Presidencia de Quito, Quito: Editorial AbyaYala.

Dueñas Carmen, 2010, «Los viajes de los indios de Portoviejo a la Corte española: conflictos interétnicos y territoriales», Procesos. Revista Ecuatoriana de Historia, n. ${ }^{\circ} 31$ (I semestre), 5-24, disponible en http://revistaprocesos.ec/ojs/index.php/ojs/article/view/114/203

Fabbri Paolo \& Aurelia Marcarino, 2002, "El discurso político", Designis, n. ${ }^{\circ} 2,17-32$, disponible en https://ddd.uab.cat/pub/designis/designis_ a2002m4n2/designis_a2002n2p17.pdf

Macías Barrés David, 2014, "Patrimonio cultural y lingüístico: El montubio y el amorfino", HISAL. Histoire(s) de l'Amérique latine, vol. 10, disponible en http://www.hisal.org/?journal=revue\&page=article\&op =view\&path\%5B\%5D=Macias2014 
Macías Barrés David, 2019, "Políticas estatales e identidades en el Ecuador: etnicidades de la Costa y 'reindigenización'"', Crisol, n. ${ }^{\circ} 6$, p. 1-16, disponible en http://crisol.parisnanterre.fr/index.php/crisol/article/view/152/134

Macías Barrés David, en prensa, "El cholo más allá de la literatura: discurso e identidad", Actas del Congreso Anual de Ecuatorianistas 2019.

Palma Neptalí, 2018a, "Pueblo cholo busca su inclusión en la Constitución", El Universo, disponible en https://www.eluniverso.com/noticias/2018/06/04/nota/6792824/pueblo-cholo-busca-su-inclusion-constitucion

Palma Neptalí, 2018b, "Pueblo cholo del Ecuador trata su reconocimiento en la Constitución", El Universo, disponible en https://www.eluniverso.com/ noticias/2018/05/30/nota/6783458/pueblo-cholo-trata-su-reconocimiento-constitucion

Rodríguez Cruz Marta, 2018, "Construir la interculturalidad. Políticas educativas, diversidad cultural y desigualdad en Ecuador", Íconos. Revista de Ciencias Sociales, n. ${ }^{\circ}$ 60, 217-236, disponible en http://scielo.senescyt. gob.ec/scielo.php?script =sci_arttext\&pid=S1390-12492018000100217

Roitman Karem \& Oviedo Alexis, 2017, "Mestizo racism in Ecuador", Ethnic and Racial Studies, n. ${ }^{\circ}$ 40(15), 2768-2786, disponible en https://www. tandfonline.com/doi/abs/10.1080/01419870.2016.1260749

Sinardet Emmanuelle, 2010, "¿Describir o inventar la tradición? La construcción de una figura mítica del cholo en Don Goyo (1933) de Demetrio Aguilera Malta" en Guiraud Michèle (éd.), Fêtes et traditions dans le monde luso-hispanophone. Mélanges en l'honneur de Nicole Fourtané, Nancy: Presses Universitaires de Nancy, 157-170.

Smith Kimbra, 2015, Practically invisible: Coastal Ecuador, tourism, and the politics of authenticity, Nashville: Vanderbilt University Press. 